\title{
1995920965
}

\section{Fuzzy Logic Techniques for Rendezvous and Docking of Two Geostationary Satellites}

\author{
Guillermo Ortega \\ European Space Agency \\ European Space Operations Centre \\ D-64276 Darmstadt, Germany \\ e-mail: g.ortega@ieee.org
}

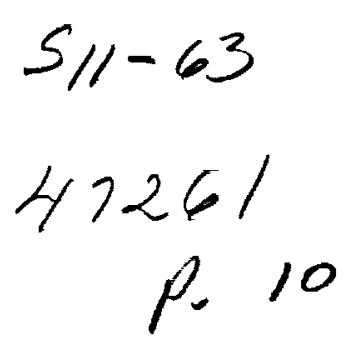

\section{ABSTRACT}

Large assemblings in space require the ability to manage Rendezvous and Docking operations. In future these techniques will be required for the gradual build up of big telecommunication platforms in the geostationary orbit.

The paper discusses the use of Fuzzy Logic to model and implement a control system for the docking/berthing of two satellites in geostationary orbit. The system mounted in a chaser vehicle determines the actual state of both satellites and generates torques to execute maneuvers to establish the structural latching. The paper describes the proximity operations to collocate the two satellites in the same orbital window, the Fuzzy guidance and navigation of the chaser approaching the target and the final Fuzzy berthing.

The Fuzzy Logic system represents a Knowledge Based Controller that realizes the close loop operations autonomously replacing the conventional control algorithms. The goal is to produce smooth control actions in the proximity of the target and during the docking to avoid disturbance torques in the final assembly orbit.

The knowledge of the Fuzzy controller consists of a data base of rules and the definitions of the fuzzy sets. The knowledge of an experienced spacecraft controller is captured into a set of rules forming the Rules Data Base.

\section{INTRODUCTION}

Since several years ago the number of spacecrafts in the geostationary orbit has increased considerably. Large satellites with longer life times are now the trends in the market. A big part of this development is due to commercial telecommunications companies with a growth on circuits demand of approximate $10 \%$ per year.

For the time being the satisfaction of this demand is fulfilled by a progressive tightening of the East-West deadband and the construction of complex clusters with collision avoidance strategies. But the concept of satellite cluster is only attractive if the functioning of the set of spacecrafts in the same orbital window is seen by the user as only one payload.

Current practice for orbital windows in GEO assumes a square of about $\pm 0.1^{\circ}$ in latitude and longitude but this is gradually being shrunk to $\pm 0.05^{\circ}$. This demand in orbital space has a big impact on spacecraft design, ground station design and station keeping operations; the spacecraft must incorporate more efficient propulsion systems and satellite-to-satellite tracking devices (ESA 10035), the ground station has to increase in complexity to allow higher accuracy in 
orbit determination and operations have to become more complex for inclination and eccentricity maintenance. Some solutions arise: satellite clusters (with heavy workload in ground operations or autonomous station keeping) or the gradual assembling of big platforms in orbit. The first technique requires orbit determination methods on board the satellite (where ground operations workload is considerably reduced) or on ground. The second requires the ability to master rendezvous and docking operations but once the assembly is formed the control remains purely conventional.

The advantages of a big platform formed by the joining of several pieces instead of a "dancing" cluster of satellites can be listed as follows:

1. reduction to zero the risk of collisions.

2. reduction in ground station workload.

3. possibility to add as many pieces as desired increasing by far the capacity of the initial payload.

On the other hand the satellites must to be constructed with docking mechanisms and autonomous control systems.

\section{RendezVOUS MISSION DESIGN}

Among the main three techniques used for a chaser approaching a target (R-bar, V-bar, inertial) the Vbar is the most common used (Leonard 89) for its stability properties. With this technique less propellant than R-bar is required but plume impingement problems appear. Here only V-bar option will be considered. For a mission of this type the following assumptions are made:

- the target: it is a geostationary communications satellite. The target is passive and has the necessary mechanical-electrical elements for the docking of the chaser. It is maintained in the centre of its orbital window within a specified inclination and eccentricity. The target is three-axis stabilized via a double-gimbaled, bias momentum control system during the complete rendezvous mission.

- the chaser: it is another geostationary communications satellite with identical shape and mass. The chaser has a control system that allows the rendezvous and soft docking with the target in an autonomous form (no man-in-the-loop capabilities are considered). If the rendezvous fails the chaser returns to a safety position. The chaser is approaching the target using the V-bar technique where the docking axis is along the velocity vector.

- the environment: the chosen reference coordinate system is the Local Vertical, Local Horizontal (LVLH) (fig. 1). That is: $+X$ in the direction of target flight, $+Z$ in the direction of center of Earth and $+Y$ towards the Earth south pole. The orbital

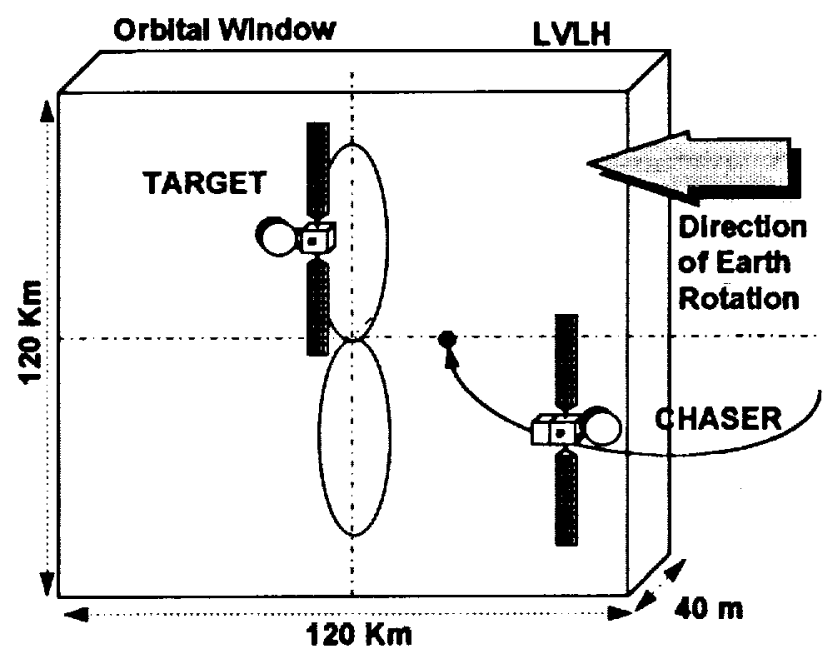

Figure 1. Final appraach and injection into orbital window

window measures are $\pm 0.1^{\circ}$ in longitude and latitude. This window is maintained by means of ground operations with a full antenna coverage in all occasions. The control centre could be located in ESOC (Germany) having an antenna which monitors the first part of the operation.

Under these assumptions the type of mission design for an assembling in GEO of two satellites will not be different from the conventional design during the launch, transfer and drift orbits and the station acquisition phase (Pocha 87). The difference resides in the guidance and navigation of the chaser approaching the target and the final docking.

\section{Launch and GTO Orbit}

The launch can be performed from Kourou Space Centre in French Guyana using an Ariane 4 expendable three-stage rocket. Using this rocket the inclination of the transfer orbit is $7^{\circ}$. At the apogee of the transfer orbit the apogee motor is fired acquiring a 
near geosynchronous orbit (the drift orbit).

\section{Drift Orbit and Station Acquisition}

During the drift orbit the chaser acquires its operational three-axis stabilized attitude pointing involving de-spin, Sun acquisition and Earth acquisition. For this orbit a set of 4 ESA ground stations to track the target can be assumed.

Once the satellite has acquired its operational attitude stabilization it must be placed closed to the target orbital longitude and with a specific orbit inclination. This operation is accomplished using ground tracking support. In this case only one station is involved.

\section{Rendezvous \& Docking Operations}

These are defined as the set of operations to close up and dock two spacecrafts. In a typical mission they

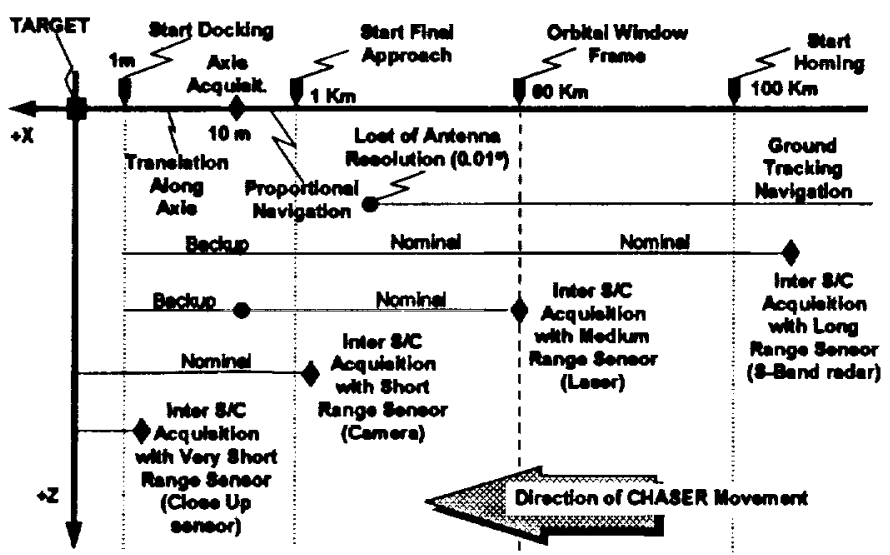

Figure 2. Rendezvous sequence timeline

comprise (fig 2): homing, final approach, docking and structural latching (Wohlke 92, Pairot 92).

\section{Homing}

This phase starts with the target presence acquisition by the large range sensor (S-band radar) mounted in the chaser. That happens approximately at $-100 \mathrm{Km}$ (LVLH system) behind and slightly below the target. In this phase although the ground station supplies navigation data, the radar works in back-up mode giving $R, A z$ and $E l$ of the target. During this phase only translation movement of both target and chaser is considered.

At $-60 \mathrm{Km}$ the chaser crosses the orbital window and in that moment the medium range sensor (laser) locks-on the target. During this part the laser sensor and radar sensor are nominal. Now the attitude pointing of the chaser starts to be important.

The homing phase extends to a distance of $-1 \mathrm{Km}$ (behind the target). In that moment another phase starts.

\section{Final Approach}

This phase comprises the close up of the chaser from $-1 \mathrm{Km}$ to $-1 \mathrm{~m}$. During this time the short range sensor (camera) localizes a specific mark in the target (Ho 93). In this phase the camera functions in nominal mode whereas the laser and the radar are in backup mode.

\section{Docking}

This phase starts at $-1 \mathrm{~m}$ from the target and ends just a few centimeters from it, before the latching. Four close up sensors mounted in a cruciform way in the chaser west platform side allow the fine docking.

\section{Latching}

Four latches mounted clockwise to the close up sensors will fit into four handles that close when the proximity operations are finished (Fehse 85). In this phase the sensors and the camera operate in parallel.

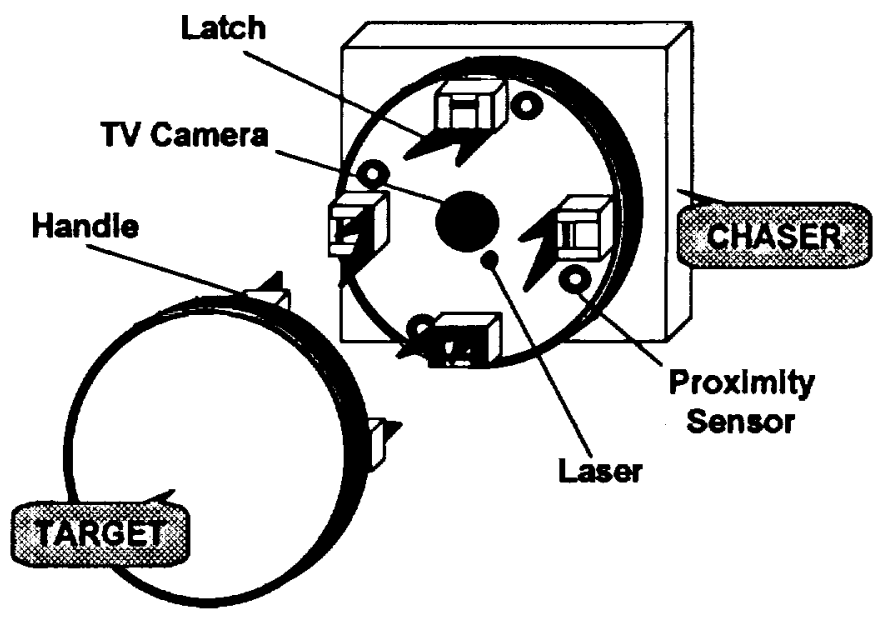

Figure 3. Docking mechanism

The laser and radar data are not considered (fig. 3). 


\section{THE PHYSICS OF THE SYSTEM}

The translational motion of a spacecraft system in circular orbit can be described using the ClohessyWiltshire equations. Those are linear differential equations with time constant coefficients which describe the movement of two masses in a circular orbit around a third object. They were programmed in the rendezvous guidance computer used in the Gemini mission (1962) and still provide short-range maneuver computation for the Shuttle. Nowadays the range of validity of these equations have been extended by introducing special coelliptic coordinates to generalize the LVLH reference system.

The Clohessy-Wiltshire equations have as input the initial position and velocity of the chaser. The output is the position and velocity of the chaser after a time interval. To apply these equations the two rotating bodies must have a small mass in comparison to the non-rotating body. In addition the target is located in the origin of the rotating coordinate system. The position and velocity of the chaser are given in this LVLH reference system.

$$
\ddot{r_{2}}-\ddot{r_{1}}=\frac{\mu}{r_{1}^{3}}\left[r_{1}-\frac{r_{1}^{3}}{r_{2}^{3}} r_{2}\right]+f
$$

where $r_{1}, r_{2}$ are the distances of target and chaser from the Earth centre, $\mu$ is the product $G^{*} M_{\text {ourth }}$ and $f$ is the perturbing force. This equation linearised and written in terms of Cartesian coordinates (LVLH system) gives:

$$
\begin{aligned}
& \ddot{x}-2 \omega \dot{z}=f_{x} \\
& \ddot{y}+\omega^{2} y=f_{3} \\
& \ddot{z}+2 \omega \dot{x}-3 \omega^{2} z=f_{z}
\end{aligned}
$$

These equations are not solvable in general but in some special cases it is possible to derive an analytical solution. If the extemal forces are zero $(f=0)$ the equations can be applied to a rendezvous in high LEO orbits (Shuttle, Soyuz-MIR) giving excellent results. (Malyshev 94, Brown 94). For a rendezvous in low LEO the force $f$ is the atmospheric drag, etc.

In the geostationary case several forces (apart from the Earth gravity) perturb the orbit of satellites: the Moon, Sun, Earth triaxiality, solar wind, etc. The Moon-Sun combined effect causes the orbit inclina- tion to grow $0.85^{\circ} \mathrm{yr}^{-1}$ in average. The Earth's oblateness causes a precession of the ascending node of about $4.9^{\circ} \mathrm{yz}^{-1}$. This effect is noticeable for non inclined orbits but it is negligible for orbits with $0^{\circ}$ inclination. The Earth triaxiality causes a longitudinal acceleration towards GEO points at $79^{\circ} \mathrm{E}$ and $107.6^{\circ} \mathrm{W}$. An average value in acceleration of $0.001^{\circ}$ per day ${ }^{2}$ towards these points is typical. Finally the solar radiation pressure varies as the inverse square of the distance from the Sun. This force depends on the type of spacecraft surface and can be estimated as:

$$
F_{\mathrm{SP}}=k * A * 1.6 * \cos i
$$

The magnitude of this force along pitch axis is $10^{-4}$ $, \cos \omega_{\text {geo }}, \mathrm{t} \mathrm{Nm}$ and the magnitude along roll and yaw axis is $10^{-5} \cdot \cos \omega_{\text {geo }}$.t $\mathrm{Nm}$.

Once the chaser is close to the target during the final approach phase the force of the Earth gravity can be considered uniform. In this case the reference system becomes inertial body reference system and the equations become:

$$
\begin{aligned}
& m_{\text {chaver }} \bar{x}=\mathrm{f}_{x} \\
& m_{\text {chaver }} \bar{j}=\mathrm{f}_{y} \\
& m_{\text {chaver }} \bar{z}=\mathrm{f}_{z}
\end{aligned}
$$

where again $f$ is the perturbing force and $x, y, z$ are

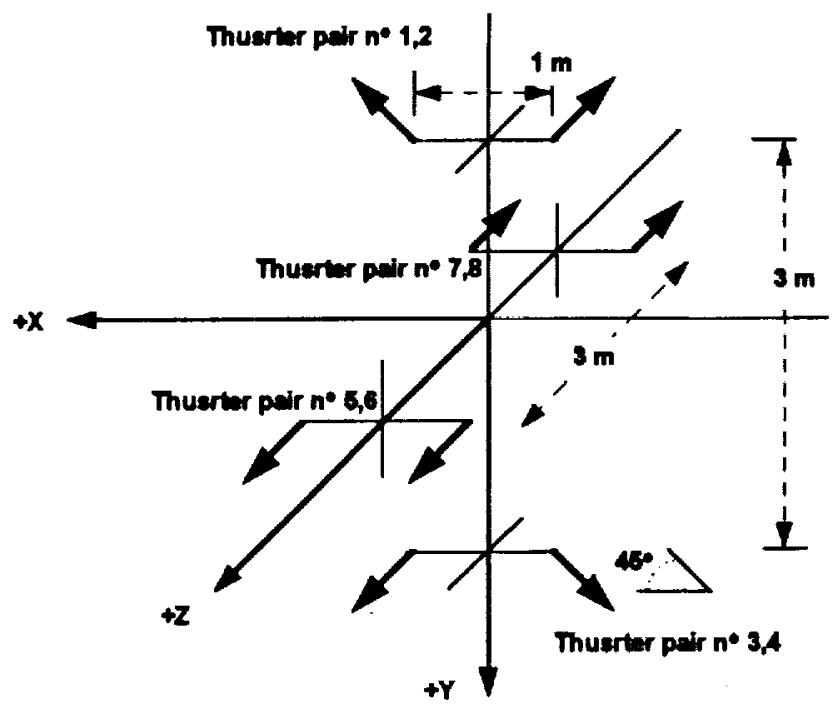

Figure 4. Thrust location in chaser 
the chaser coordinates with respect to the LVLH centre. The target system physics of movement can be described using body coordinate equations as well.

The translation of both vehicles is realized by means of thrust impulses. The thruster system consists of 8 pairs localized at four surfaces as shown in (fig. 4). Pairs $(5,6)$ will impulse the satellite in the $-X$ direction and pairs $(7,8)$ in $+X$ direction, etc.

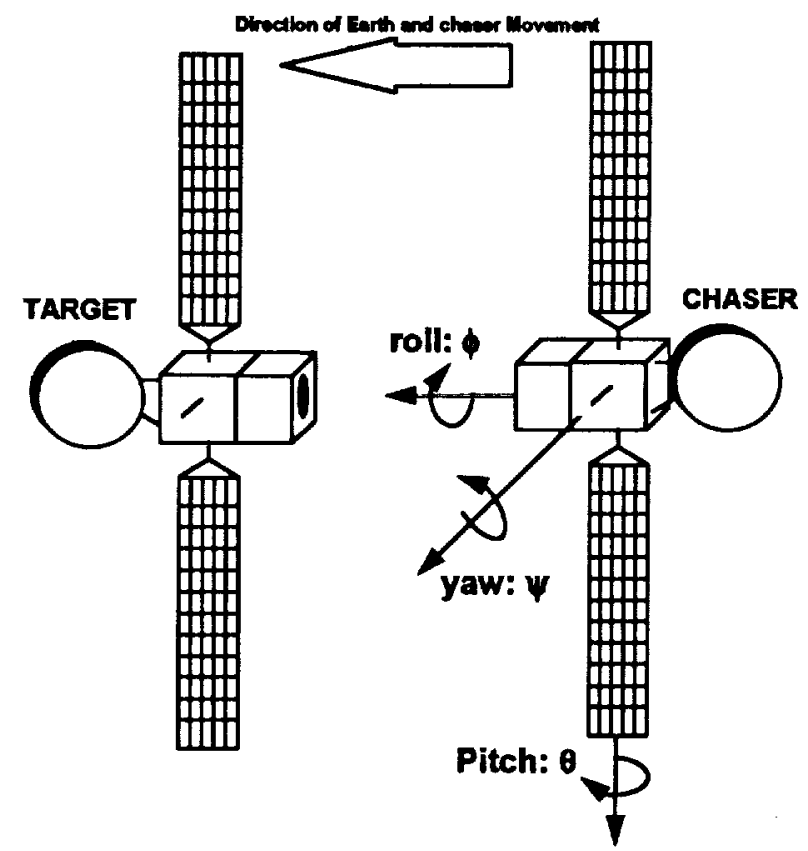

Figure 5. Final closing body coordinate system

The attitude control of both chaser and target is designed as a three axis stabilized, double gimbaled, bias momemtum control system (Kaplan 76, Wertz 78). The corresponding Euler equation is

$$
\left(\mathbf{F}_{d}+\mathbf{F}_{g g}\right)_{s^{\prime}}=\left[\frac{d \mathbf{h}}{d t}\right]_{s}+\mathbf{w}_{s s^{\prime}} \times \mathbf{h}_{s}
$$

where $S^{\prime}$ is a fixed reference system with origin in the centre of satellite mass and $S$ is a reference system with the same origin that rotates with the satellite. $S$ ' and $S$ are defined as the LVLH system. $F_{d}$ is the disturbance force (solar pressure, thruster misalignment, etc.) and $F_{a g}$ is the force due to gravity gradient. $w_{z z^{\prime}}$ is the angular velocity of $S$ respect to $S^{\prime}$ and $h$ is the total angular momentum of the spacecraft (body + gimbaled wheel).
Pitch equation is simple and decoupled from yaw and roll assuming a symmetric satellite

$$
F_{\text {क }}=I_{3} \ddot{\theta}+\dot{h} \text {, }
$$

but yaw and roll equations are coupled:

$$
\begin{aligned}
& F_{\alpha x}=1_{x} \ddot{\phi}+\omega_{s s} h_{w} \phi+h_{w} \dot{\psi}+\dot{h}_{x}-\omega_{s s} h_{z} \\
& F_{d z}=l_{z} \ddot{\psi}+\omega_{s s} h_{w} \psi-h_{w} \dot{\phi}+\dot{h}_{z}-\omega_{s s} h_{x}
\end{aligned}
$$

where $l_{x} l_{3}, l_{z}$ are the principal moments of inertia and the control torques $\left(\dot{h}_{x}, \dot{h}_{y}, \dot{h}_{z}\right)$ are produced through gimbals defections (fig. 5 ).

\section{FUZZY CONTROL: SYSTEM MODEL}

Figure 6 shows the proposed Guidance, Navigation and Control System (GNC). The navigation block calculates the actual state of both spacecrafts and their relative measurements. The guidance and control blocks are together in the figure. The guidance part calculates the future state of the spacecraft to achieve

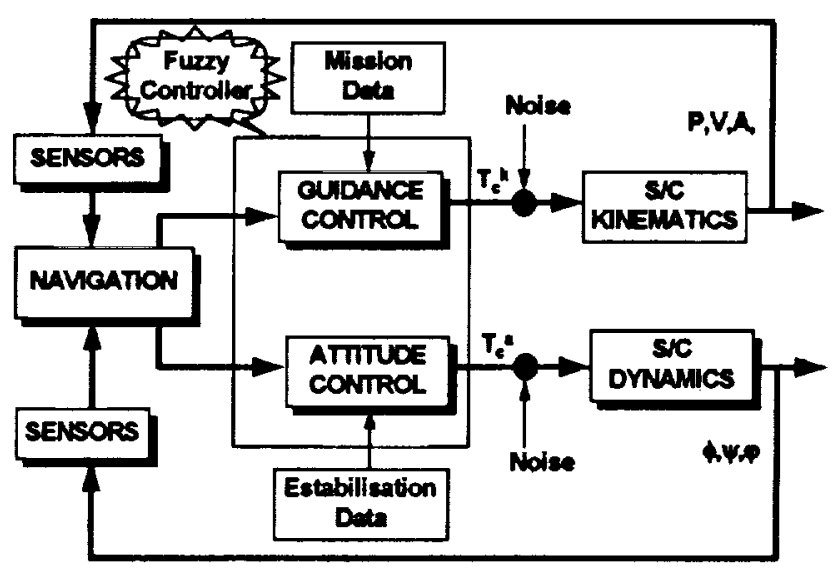

Figure 6. Guidance, navigation and control system

the desired trajectory and the control part calculates the desired control torques to achieve this trajectory.

It must be noticed that the guidance and control (G\&C) system is divided in two parts: the G\&C of the translation movement and the G\&C of the rotational movement. For this study both movements are supposed to be de-coupled and the influence of one on the other is considered negligible. However we will see that the attitude motion influence on the G\&C 
part of the translation motion.

\section{Controller Implementation}

The fuzzy controller (FC) must substitute the guidance and control parts. The goal of the FC is to be able to generate the control signals derived from the sensor measurements ( $\operatorname{Lee} 90)$. The steps involved in the construction of this FC are the following:

1. define input and output variables.

2. define universe of discourse for all variables.

3. define the Rule Data Base (RDB).

4. define the Inference Engine

Once the FC is constructed it must be coded and included in the simulation.

For all the four previous points a fundamental step in the implementation of the FC is to capture the knowledge of an expert. This knowledge must be applied to guide and to control the vehicle. The expert will help defining the way to determine the state of the targetchaser system and the way to use the commands for each situation. The inference engine can be an approximate reasoning kemel based on already proposed systems (Buckley 92, King 94).

\section{Construction of IFS and OFS}

In this case the input variables are the sensor measurements (positions, velocities, etc.) and the output variables are the firing of thrust (thrust position and time of fire) and the attitude angles and rates. The input variables will be represented in the Input Fuzzy Sets (IFS) system and the output variables will be represented in the Output Fuzzy Set (OFS) system (Drianov 93).

For the translation movement it is simpler to use input variables in polar coordinates rather than Cartesian: an aircraft pilot is able to measure azimut and elevation angles and rates and distances and distances rates. The rotational movement can be represented by the attitude angles and angles rates. The output for a human controller could be the deflection of a joystick to fire a particular thruster and the defection time for acceleration controlled devices or the amount of pulses in pulse control devices.

\section{Defining the control variables}

For the translation movement of the chaser the input variables of the $\mathrm{FC}$ system will be azimut $(a)$, eleva- tion $(e)$, azimut rate $(\dot{a})$, elevation rate $(\dot{e})$, range (r) and range rate $(r)$. For the rotational part the input variables will be pitch $(\theta)$, pitch rate $(\dot{\theta})$, roll $(\phi)$, roll rate $(\dot{\phi})$, yaw $(\psi)$ and yaw rate $(\dot{\psi})$. The output variables will be the amount of firing time ( $\mathrm{ft}$ ) and the position of fired thruster (pt).

The universes of discourse of these variables are as follows:

$$
\begin{aligned}
& a \in[-\pi, \pi] \text { in rad, } a \in[-2,2] \text { in } \% / \mathrm{sec} \\
& e \in[-\pi, \pi] \text { in } \mathrm{rad}, \dot{e} \in[-2,2] \mathrm{in} \% \mathrm{sec} \text {, } \\
& r \in\left[0, R_{\text {mex }}\right] \text { in } \mathrm{Km}, \quad r \in[-10,10] \text { in } \mathrm{m} / \mathrm{s} \\
& \theta \in[-\pi, \pi] \text { in } \mathrm{rad}, \dot{\theta} \in[-0.5,0.5] \mathrm{in} \% \mathrm{sec} \\
& \phi \in[-\pi, \pi] \text { in rad, } \dot{\phi} \in[-0.5,0.5] \text { in } \% / \mathrm{sec} \\
& \psi \in[-\pi, \pi] \text { in } \mathrm{rad}, \psi \in[-0.5,0.5] \text { in } \% \text { sec } \\
& \text { Bum } \in[-10,10] \text { in } \mathrm{dcm} / \mathrm{sec}
\end{aligned}
$$

\section{Defining the Fuzzy Sets}

The fuzzy subdivisions of the universe of discourse of any variable are called the fuzzy sets. Every 'partial'

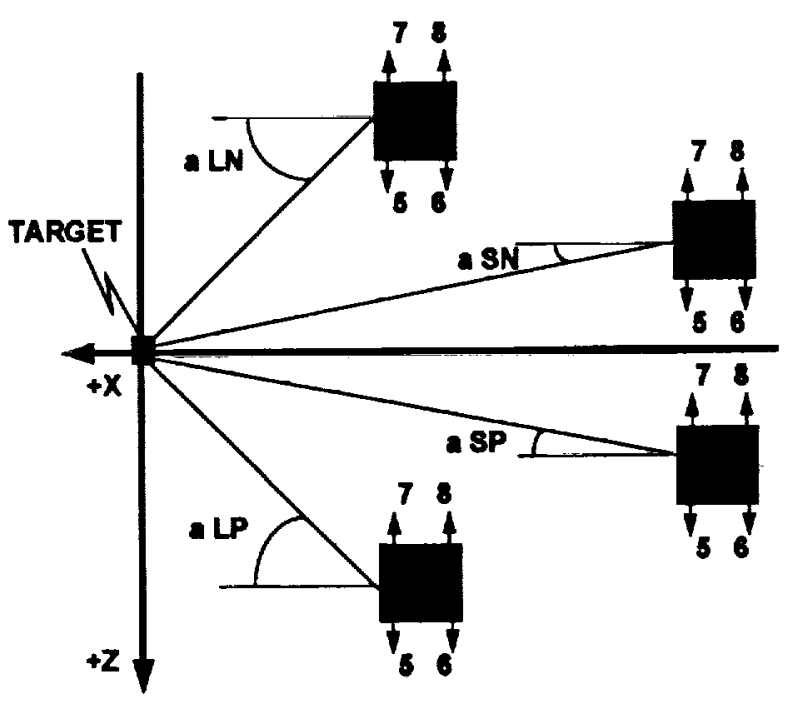

Figure 7. Azimut fuzzy set selection

value of each variable can belong to more than one set.

For this study the following sets are assumed: 
- for azimut, azimut rate, elevation, elevation rate and range rate the fuzzy sets are Small Negative (SN), Small Positive (SP), Large Negative (LN) and Large Positive (LP) (fig. 7).

* for range the possible fuzzy sets are Small (S) and Large (L) as no more distinctions are needed.

* for roll, pitch, yaw and its derivatives the fuzzy sets are Small Negative (SN), Small Positive (SP), Large Negative (LN) and Large Positive (LP)

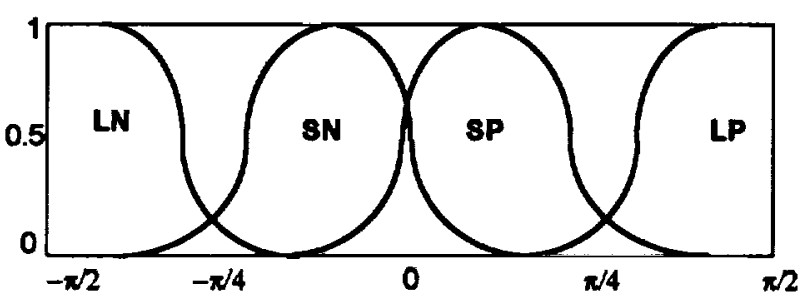

Figure 8. Fuzzy representation for azimut

Special attention must be put when creating the size and type for the fuzzy membership functions. Depending on the position along the universe of discourse axis, the control actions will be more or less smooth. A rule for relative good control states that the functions should overlap $25 \%$. The profile of the function can be trapezoid, bell shaped, gaussian, etc. A first approach in the definition of the membership functions for the problem of the rendezvous is shown in figure 8.

The azimut of the chaser (movement along $\mathrm{X}$ axis) is subdivided in the four fuzzy sets $(L N, S N, S P, L P)$. For small angles $(a \in[-\pi / 4, \pi / 4])$ the exponential gaussian shapes overlap a little bit more than $25 \%$ trying to smooth thruster actions within these limits.

\section{Rules Data Base}

The rules data base is the kemel of the knowledge base controller (KBC). Thanks to this data base the FC will incorporate an experience which can only be realized in the corresponding analytic model by means of manual operations. In this case the KBC implements the close loop control actions substituting the operator (Tolk 94).

\section{Capturing the knowledge}

Here the expert tells us the following:
$\Rightarrow$ the control of each axis is carried out in an independent manner: firings in $Z$ direction control azimut and firings in $Y$ direction control elevation. The firings over each axis are calculated taking into account angles and its derivatives.

$\Rightarrow$ the control in azimut and elevation is not symmetric: due to the Clohessy-Wiltshire equations of movement firings in $\mathrm{X}$ direction will 'elevate' the chaser in its path towards the target. It is necessary to realize compensation firings in the $+Z$ direction. However, the control in elevation is symmetric with respect to the $+X$ axis.

$\Rightarrow$ the tendency of human controllers is to make azimut and elevation equal to $0^{\circ}$ during the final approach phase (typically at -10 or $-20 \mathrm{~m}$ of the target) to be able to reduce control workload and focus on fine range rate control. This scheme complies with an intuitive proportional navigation guidance towards the point of starting axis translation.

$\Rightarrow$ the amount and size of the firings depend proportionally on the distance: different firing strategies must be followed depending on the distance to the target. When the chaser is in homing phase the amount of firings is low (typically 1 every $5 \mathrm{~min}$ utes) and the size is big. In final approach phase the firings amount starts to increase ( 1 every minute) but the size gets small. During docking the firings are frequent with very small size.

$\Rightarrow$ attitude angles and angles rates are controlled during the translation along axis phase.

\section{Constructing the knowledge data base}

The rules data base are given in the following form:

if ANTECEDENT then CONSEQUENT

where ANTECEDENT and CONSEQUENT are any composition of statements (and, or, not, etc). The statements contain declarations of associations of fuzzy variables to fuzzy sets (fig. 9).

These rules are grouped in two categories and every category contains several groups of control. One category corresponds to a long distance between target and chaser and the other corresponds to short distance between target and chaser. This distinction allows to fire different sets of rules belonging to translation or rotational movement: during long distances ( $r$ is large) no rule of rotational movement is fired. When the chaser is getting closer to the target 
the relative attitude of both vehicles starts to be im-
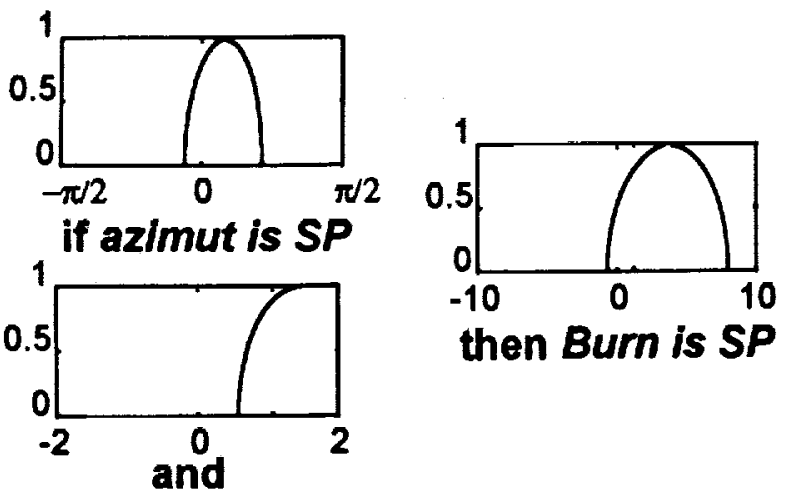

azimut rate is $L P$

Figure 9. Azimut rule

portant.

For each category the rules are grouped depending on the control action they generate: for the translational movement there are rules for controlling azimut, rules for controlling elevation and rules for controlling distance; for the rotational movement there are rules for controlling pitch, rules for controlling roll and rules for controlling yaw;

\section{SIMULATIONS}

For the simulations, the size of the satellites is assumed to be about $15 \mathrm{~m}$ high from the extreme ends of the solar panels and the body size is about $3 \mathrm{~m}^{3}$ (e.g. ESA ECS satellite). The mass is $1000 \mathrm{Kgr}$. The matrix of moments of inertia is diagonal. The thruster misalignment torque is of an order of magnitude less than the solar pressure. The attitude pointing requirements for the soft docking are $0.005^{\circ}$ in pitch and roll and $0.4^{\circ}$ in yaw. The docking velocity is 1 $\mathrm{cm} / \mathrm{s}$.

The plant description is simulated using the ClohessyWiltshire equations for the chaser influenced by a constant force in the - $Y$ direction (Moon-Sun attraction) during the first part of the rendezvous. During the final approach phase the reference system is inertial body and the equations get linear. The target translation is simulated with a constant force in the same direction. The attitude dynamics of both space- crafts are simulated using the Euler equations with gravity gradient and solar pressure perturbations.

The FC controller is implemented in the following way:

- The control variables together with their universes of discourse are as described before.

- The shape of the membership functions is an exponential gaussian function.

- The rule data base is composed of 32 rules (20 for translation and 12 for rotation).

- The inference engine is programmed using the Mamdani's Min-Max mechanism (Mamdani 74); the AND operator is chosen as the minimum of two weight antecedents instead of its multiplication.

- The defuzzyfication strategy used is the centre of gravity computation.

The pseudocode of the simulation is as follows:

\section{initialize all;}

create rules for the rules data base;

step $=1$

big_loop

for $t=1$ to time to fire again

draw positions \& velocities;

chaser kinematics;

target kinematics;

chaser attitude dynamics;

target attitude dymamics;

sensor measurements;

store everything for drawing;

end for:

Fuzzy_control_computations;

if $R=0$ then out

else step $=$ step +1 ;

end big_loop;

Figure 10 shows a simulation run. The chaser was initially located in the coordinates $(-1,0,1)$ behind the target at $-1 \mathrm{Km}$. The firing of thrusters try to close the chaser to the docking axis as soon as possible. This is practically achieved at $-400 \mathrm{~m}$. The translation along the axis occurred at $-10 \mathrm{~m}$. The azimut of the chaser decayed from $45^{\circ}$ to $10^{\circ}$ in a nearly exponential curve. From $10^{\circ}$ to $0^{\circ}$ the azimut waved up and down. This movement corresponds to the different trials to align with the docking axis.

Figures $11 \mathrm{a}$ and $11 \mathrm{~b}$ show another simulation run. This time the chaser was initially located in the coor- 


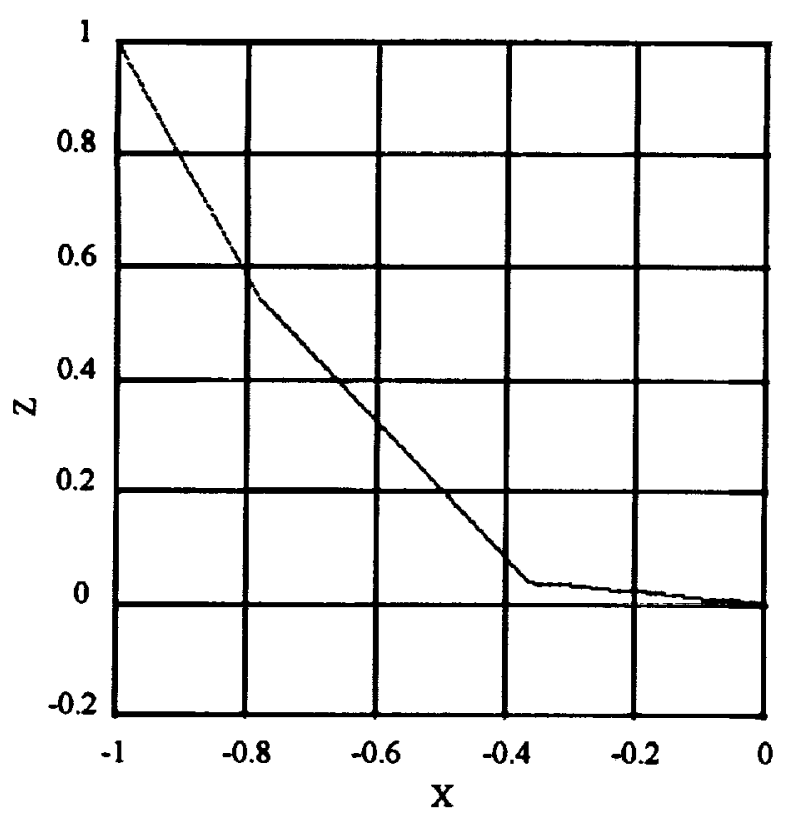

Figure 10. Simulation run (in-plane motion;

dinates $(-0.05,-0.05,0.05)$ behind the target at $-500 \mathrm{~m}$ and below the target at $-500 \mathrm{~m}$ as well. The firing of thrusters, for the in-plane motion (fig. 11a), try to

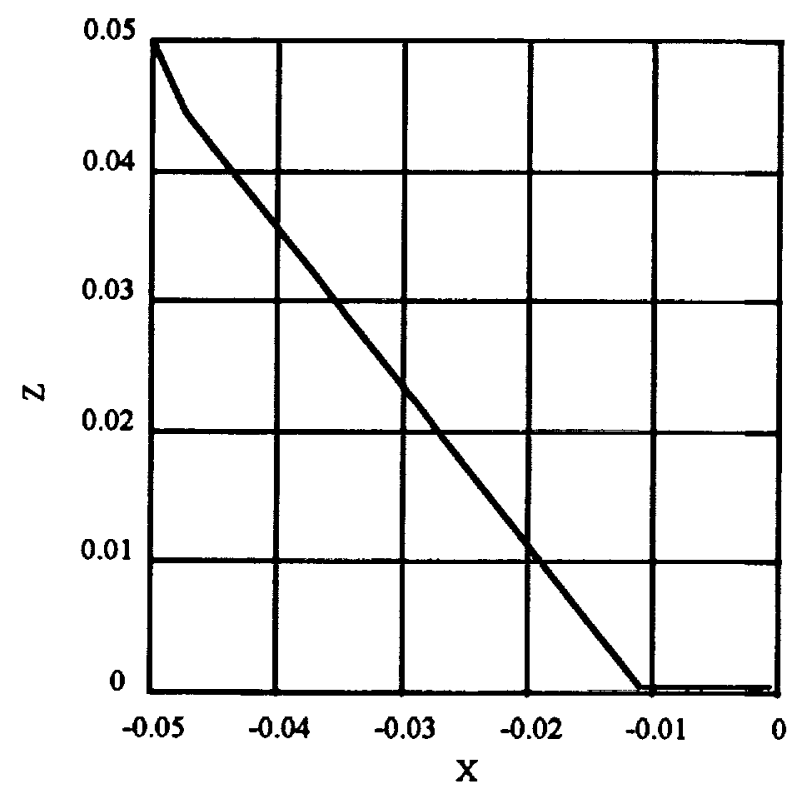

Figure 1la. Simulation run (azimut) in-plane motion

close the chaser to the docking axis as soon as possible. For the out-of-plane motion (fig. 11b) the firings were almost linear (because the angular velocity of the orbit is very low). The translation along the axis occurred at $-11 \mathrm{~m}$. The azimut of the chaser decayed again from $45^{\circ}$ to $10^{\circ}$ in a nearly exponential curve. From $10^{\circ}$ to $0^{\circ}$ the azimut waved up and down as well. The elevation of the chaser decayed from $45^{\circ}$ to $0^{\circ}$ in a nearly linear curve. From $10^{\circ}$ to $0^{\circ}$ the elevation did not waved up and down.

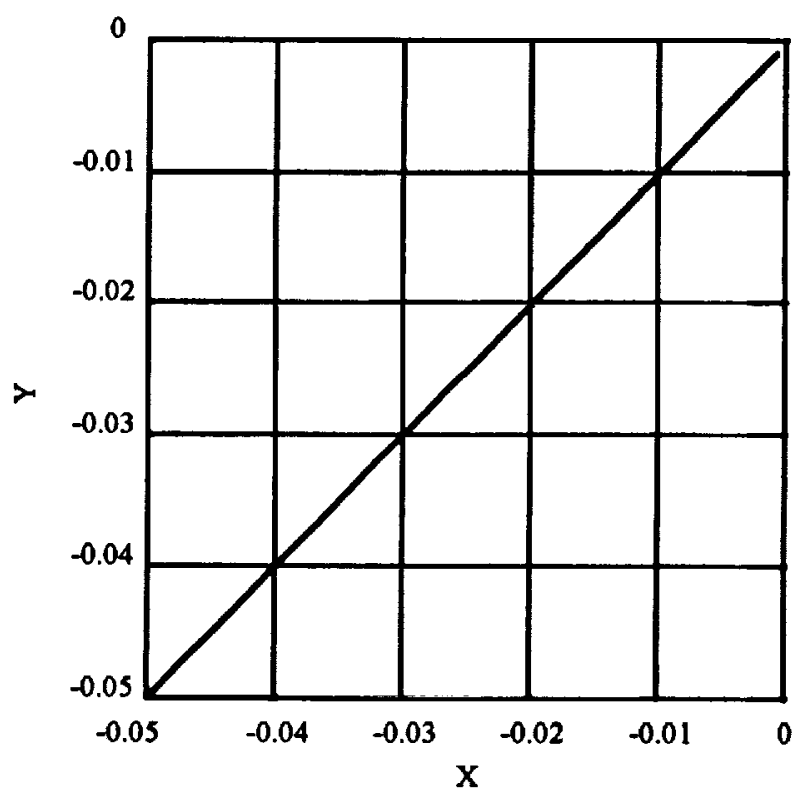

Figure 11b. Simulation run (elevation) out-of-plane motion

Simulations were developed on a PC compatible 486/66DX2 portable type computer. The code was written in MATLAB $4.0^{\circ}$ for Microsoft Windows ${ }^{\mathrm{TM}}$. The fuzzy inference engine with approximate reasoning was implemented using FISMAT, the Fuzzy Inference Systems toolboox for MATLAB developed by A. Lofti. FISMAT provided fuzzy logic operations and different methods of approximate reasoning.

\section{CONTINGENCIES}

Contingency situations can happen when the docking velocity is too high, the docking axis is not in perfect alignment with the V-bar axis or when the attitude of both satellites differ in an angle bigger than the one specified for the latching mechanism.

Easily, the FC can be extended to handle these situations. For an hazardous impact the thrust pair $(1,3)$ must be used to produce an exponential breaking 
during the translation along axis. This situation was taken into account with the rule

if range rate is $L$ then Burn $(1,3)$ is $S N$

For the situation with the docking axis not in perfect alignment with the $\mathrm{V}$-bar axis a pseudo proportional navigation towards the starting point of the axis translation was implemented with the rule

if $a$ is $L P$ and a rate is $L P$ then Burn $(5,6)$ is $L P$

Finally when attitude angles are out of range of the specifications of the latching mechanism some corrective measures can be taken during the translation along-axis phase.

\section{Conclusions}

Up to date, servicing is greatly dependent of human operations in space. Therefore, the on-orbit accessibility factors of the satellite to be serviced is a primary consideration. Support capabilities for human presence in satellite maintenance tasks in GEO will not be widely available until the first decade of the 21 st century (Waltz 90). In the meantime autonomous rendezvous and docking arise as a good alternative.

Fuzzy logic emulates the behavior of human operators for complex control tasks. A fuzzy logic controller embedded in a guidance, navigation and control system of a spacecraft can realize autonomously the close loop operations replacing the conventional crisp control algorithms. The fuzzy controller produces soft control actions in the proximity of the target and during the docking to avoid disturbance torques in the final assembly.

Fuzzy controllers can be programmed, tested and qualified for flight (Daley 85,87 ). Its rule data base can be constructed with the help of an expert and refined in simulations. Fuzzy controllers are easily reconfigurabled for different type of missions and their performance is robust to changes.

\section{REFERENCES}

Study on Intersatellite Tracking for Co-location (ESA/10035/92/D/CS)

An Introduction to Mission Design for Geostationary Satellites (J.J. Pocha; D. Reidel Publishing Company 1987, ISBN 90-277-2497-2).

Rendezvous and Berthing between Columbus Free Flying Laboratory and Space Station Freedom (W. Wohlke; Automated Control in Aerospace 1992).
Development of an Autonomous Onboard Control System for Rendezvous and Docking (J.M. Pairot, W. Fehse, A. Getzschmann; Automated Control in Aerospace 1992).

Rendezvous and Docking Technology Development for Future European Missions (W. Fehse; ESA Joumal 1985, vol. 9).

Modern Approaches on Guidance and Control of Russian Spacecraft (V.V. Malyshev, M.N. Krasilshikov; 2nd ESA International Conference on Spacecraft Guidance, Navigation and Control Systems, Aril.

Fuzzy Logic Application for Modeling Man-In-The-Loop Space Shuttle Proximity Operations (R. Brown, NASACR-188283).

Modern Spacecraft Dynamics and Control (M.H. Kaplan; John Wiley \& Sons 1976, ISBN 0-471-45703-5).

Spacecraft Attitude Determination and Control (J.R. Wertz; Kluwer Academic Publishers 1978).

Fuzzy Logic in Control Systems Parts I and II (Chuen Chien Lee; IEEE Transaction on Systems, Man and Cybernetics, Vol. 20, No. 2, March-April 1990).

Universal Fuzzy Controller (J.J. Buckley; Automatica: Journal of the International Federation of Automatic Control -IFAC-, Vol. 28 Number 6, November 1992).

Multivariable Fuzzy Controller Design (R.E. King, G.D. Magoulas, A.A. Stathaki; Automatica: Journal of the International Federation of Automatic Control -IFAC-, Vol. 2 Number 3, June 1994).

An Introduction to Fuzzy Control (D. Drianov, H. Hellendoorn, M. Reinfrank; Springer-Berlag 1993).

Fuzzy Logic: A Practical Approach (F.M. McNeill, E. Thro; Academic Press 1994, ISBN 0-12-485965-8 Automatic Programming of Fuzzy Controllers by Examples (A. Tolk, K. Helling; AI-Journal, June 1994).

Application of fuzzy algorithm for control of simple dynamic plant (E.H. Mamdami; Proc. IEE, 121, no. 12, December 1974).

A Justification for the Wider use of Fuzzy Logic Control Algorithms (S. Daley, K.F. Gill; Proceedings of the Institute of Mechanical Engineering, Vol. 199, No. C1, 1985). Attitude Control of a Spacecraft Using an Extended SelfOrganizing Fuzzy Logic Controller (S. Daley, K.F. Gill; Proceedings of the Institute of Mechanical Engineering, Vol. 201, No. C2, 1987)

Automatic Spacecraft Docking Using Computer VisionBased Guidance and Control Techniques (Chi-Chang J. Ho, N. Harris McClamroch; Journal of Guidance, Control and Dynamics, vol. 16, No. 2, March-April 1993).

\section{Disclaimer}

The opinions expressed in this paper belong to its author and do not necessarily correspond to the opinions of ESA as a public institution. 